\title{
Does Cortical Brain Morphology Act as a Mediator Between Childhood Trauma and Transition to Psychosis in Young Individuals at Ultra-High Risk?
}

Running title: Trauma, brain markers and transition to psychosis

Marta Rapado-Castro ${ }^{\mathrm{a}, \mathrm{b}}$, Sarah Whittle ${ }^{\mathrm{b}}$, Christos Pantelis ${ }^{\mathrm{b}}$, Andrew Thompson ${ }^{\mathrm{c}, \mathrm{d}}$, Barnaby Nelson ${ }^{\mathrm{d}, \mathrm{e}}$, Eleni P. Ganella ${ }^{\mathrm{b}, \mathrm{d}, \mathrm{e}}$, Ashleigh Lin ${ }^{\mathrm{f}}$, Renate L.E.P. Reniers ${ }^{\mathrm{g}, \mathrm{h}, \mathrm{i}}$, Patrick D. McGorry ${ }^{\mathrm{d}, \mathrm{e}}$, Alison R. Yung ${ }^{\mathrm{d}, \mathrm{e}, \mathrm{j}}$, Stephen J. Wood ${ }^{\mathrm{b}, \mathrm{d}, \mathrm{e}, \mathrm{k}}$ and Cali F. Bartholomeusz ${ }^{\mathrm{b}, \mathrm{d}, \mathrm{e} *}$

${ }^{\text {a }}$ Department of Child and Adolescent Psychiatry, Institute of Psychiatry and Mental Health, Hospital General Universitario Gregorio Marañón, School of Medicine, Universidad Complutense, IiSGM, CIBERSAM, Madrid, Spain

${ }^{\mathrm{b}}$ Melbourne Neuropsychiatry Centre, Department of Psychiatry, The University of Melbourne \& Melbourne Health, Victoria, Australia

${ }^{c}$ Division of Mental Health and Wellbeing, Warwick Medical School, University of Warwick, UK

${ }^{\mathrm{d}}$ Orygen, Parkville, Victoria, Australia

${ }^{\mathrm{e}}$ Centre for Youth Mental Health, The University of Melbourne, Parkville, Victoria, Australia

${ }^{\mathrm{f}}$ Telethon Kids Institute, University of Western Australia, Perth, Australia ${ }^{\mathrm{g}}$ Institute for Mental Health, University of Birmingham, United Kingdom

${ }^{\mathrm{h}}$ Institute of Clinical Sciences, University of Birmingham, United Kingdom

${ }^{\text {i }}$ Centre for Human Brain Health, University of Birmingham, United Kingdom

${ }^{\mathrm{j}}$ Division of Psychology and Mental Health, Faculty of Biology, Medicine and Health, University of Manchester, UK and Greater Manchester Mental Health NHS Trust, UK

${ }^{\mathrm{k}} \mathrm{School}$ of Psychology, University of Birmingham, United Kingdom

\section{*Corresponding author:}

Cali F. Bartholomeusz; Orygen, 35 Poplar Road, Parkville, Victoria, Australia, 3052

Email: cali.bartholomeusz@ orygen.org.au; Phone: +61 93422800

Word count: Abstract (250 words) and Text body (5.000 words)

Tables: 1; Figures: 2 (Supplementary Tables: 10). 


\section{ABSTRACT}

Background: Childhood trauma, particularly sexual abuse, has been associated with transition to psychosis in individuals at "ultra high-risk" (UHR). This study investigated whether the effects of various forms of childhood trauma on transition to psychosis are mediated by cortical thickness and surface area abnormalities. Methods: This prospective study used data from 62 UHR individuals from a previous (PACE 400) cohort study. At follow-up, 24 individuals had transitioned to psychosis (UHR-T) and 38 individuals had not transitioned (UHR-NT). Student-t/Mann-Whitney-U tests were performed to assess morphological differences in childhood trauma (low/high) and transition. Mediation analyses were conducted using regression and bootstrapping techniques. Results: UHR individuals with high sexual trauma histories presented with decreased cortical thickness in bilateral middle temporal gyri and the left superior frontal gyrus compared to those with low sexual trauma. Participants with high physical abuse had increased cortical thickness in the right middle frontal gyrus compared to those with low physical abuse. No differences were found for emotional abuse or physical/emotional neglect. Reduced cortical thickness in the right middle temporal gyrus and increased surface area in the right cingulate were found in UHRT compared to UHR-NT individuals. Sexual abuse had an indirect effect on transition to psychosis, where decreased cortical thickness in the right middle temporal gyrus was a mediator. Conclusions: Results suggest that childhood sexual abuse negatively impacted on cortical development of the right temporal gyrus, and this heightened the risk of transition to psychosis in our sample. Further longitudinal studies are needed to precisely understand this link. 
Key words: Cortical surface area, cortical thickness, sexual abuse, childhood trauma, psychosis, Ultra-High Risk (UHR). 


\section{INTRODUCTION}

Over two decades ago, efforts to identify individuals at risk for psychotic disorder led to the introduction of the "ultra-high risk" (UHR) criteria. Inclusion in this group requires the presence of either: (a) attenuated psychotic symptoms (APS), (b) brief limited intermittent psychotic symptoms (BLIPS), and/or (c) genetic risk (i.e., first-degree relative with a psychotic disorder or the individual has a diagnosis of schizotypal personality disorder) together with functional deterioration, in help-seeking individuals (Yung and McGorry, 1996, Yung et al., 2003, Yung et al., 2004). These criteria were developed in part, to facilitate research into aetiopathological mechanisms driving disorder progression. One aetiopathological environmental risk factor implicated in the development of psychosis is childhood trauma (Cutajar et al., 2010, Liu et al., 2015, McGrath et al., 2017, Thompson et al., 2013).

Childhood trauma has been associated with increasing risk of developing psychosis (Arseneault et al., 2011, Bendall et al., 2008, Croft et al., 2018, Cunningham et al., 2016, Kraan et al., 2018, Spauwen et al., 2006, Trotta et al., 2015, Varese et al., 2012), greater severity of attenuated psychotic symptoms (Thompson et al., 2010, Velthorst et al., 2010), persistent negative symptoms (Yung et al., 2019) poor long-term psychosocial functioning (Yung et al., 2015) and is highly prevalent (Kraan et al., 2015) in UHR cohorts. In particular, sexual abuse has been suggested to have a greater risk for development of psychosis than other forms of trauma (Bechdolf et al., 2010, Thompson et al., 2013). Currently, there is little understanding of how childhood trauma may impact vulnerability for psychosis (Isvoranu et al., 2017), especially since psychosis is not a specific outcome of childhood trauma (i.e., not all children who experience trauma later develop psychosis). Given the known detrimental 
effects of stress on the developing brain (Sowell et al., 1999, Teicher et al., 2002, Teicher et $a l ., 2012)$, it is possible that childhood trauma may lead to alterations in brain development, which in turn may play a critical role in the onset of psychosis (Mirsky and Duncan, 1986, Niendam et al., 2009, van Os et al., 2016, van Os et al., 2014).

There is firm evidence that psychotic disorders are associated with pathological changes in brain structure (for review see (Bartholomeusz et al., 2017)). Studies investigating normative structural brain development (Vijayakumar et al., 2018) suggest that the cortex globally contracts in both sexes during adolescence because of non linear decreases in cortical thickness (Blakemore, 2012, Brown and Jernigan, 2012) and volume and increases in surface area (Tamnes et al., 2017). In high-risk individuals who later develop psychosis, reductions in frontal and temporal lobe volumes are most often reported (McIntosh et al., 2011, Mechelli et al., 2011, Pantelis et al., 2003). Studies in individuals at UHR have also found cortical thickness reductions in prefrontal region (Cannon et al., 2015, Jung et al., 2011), specifically in the superior, middle frontal, orbitofrontal (Cannon et al., 2015) and anterior cingulate cortices (Jung et al., 2011, Ziermans et al., 2012), superior temporal (Jung et al., 2011, Sprooten et al., 2013) and middle temporal gyri (Sprooten et al., 2013), parietal (Jung et al., 2011, Ziermans et al., 2012), and parahippocampal regions (Jung et al., 2011, Tognin et al., 2014); and brain surface contraction in the prefrontal region (Sun et al., 2009b, Sun et al., 2009c). Further, UHR individuals who subsequently develop psychosis show more pronounced cortical thinning over time than both UHR individuals who do not develop psychosis and healthy controls, particularly in prefrontal and temporal brain regions (Cannon et al., 2015, Sun et al., 2009a, Ziermans et al., 2012). 
Consistent with the notion of childhood trauma having a detrimental impact on the developing brain, a community sample of children $(\mathrm{N}=22 M$ age=12.3) exposed to maltreatment have shown cortical thinning specifically in the superior frontal gyrus, orbitofrontal cortex and anterior cingulate, and reduced cortical surface area in the left middle temporal and lingual gyri relative to non-maltreated peers (Kelly et al., 2013). The children with documented exposure to maltreatment (i.e. rated by a social worker in relation to neglect, physical, sexual and emotional abuse) presented with significant conduct and hyperactivity problems. Authors proposed that cortical aberrations might represent markers of heightened risk for later development of psychopathology (Kelly et al., 2013). In a more recent study, youth $(\mathrm{N}=22 M$ age=17.6) with a documented history of childhood physical abuse had reduced volumes/thickness predominantly in the precuneus and precentral regions, yet they displayed increased volumes in the left inferior and middle temporal gyri (Lim et al., 2018b). Presence of sexual abuse was an exclusion criteria in this sample. These youth presented with conduct and peer problems. Discrepancies between these two studies may be due to the definition of childhood trauma or the samples being at differing neurodevelopmental stages, and it may be that enlargements are only detectable after midadolescence. In this regard, a meta analytic review including a sample of 331 individuals (56 children/adolescents and 275 adults) with a history of various forms of childhood maltreatment (including sexual, physical, and emotional abuse; neglect; witnessed domestic violence; parental verbal abuse; and harsh corporal punishment) pointed to consistent gray matter abnormalities in late-developing ventrolateral prefrontal-limbic-temporal regions (Lim et al., 2014). The authors demonstrated significantly larger gray matter volumes in the right superior frontal and left middle occipital gyri and smaller volumes in the right orbitofrontal/superior temporal and middle temporal gyrus, amygdala, insula, and 
parahippocampal gyri and in the left inferior frontal and postcentral gyri in individuals exposed to childhood maltreatment relative to control subjects (Lim et al., 2014). Gray matter abnormalities in the left postcentral and middle occipital gyri were only found for the group of older individuals with childhood trauma. These studies provide rationale for further exploration of trauma-related biological markers of psychiatric vulnerability. To our knowledge the relationship between morphological brain abnormalities and childhood trauma in relation to risk for transition to psychosis among UHR individuals has never been investigated.

The current study aimed to explore the relationship between childhood trauma and brain morphology using data from the PACE 400 Study (Nelson et al., 2013), a long-term followup study of a UHR cohort. A history of sexual trauma in the PACE 400 sample was previously found to be associated with transition to psychosis (Thompson et al., 2013) (and risk for long term poor psychosocial functioning) (Yung et al., 2015) when other risk factors were controlled for (namely duration of untreated symptoms, year of entry into the PACE clinic, baseline functioning, age, gender, educational level, baseline psychopathology and quality of life). Therefore we aimed to investigate whether the effects of various types of childhood trauma (specifically sexual abuse, physical and emotional abuse and physical and emotional neglect) on transition to psychosis were mediated by abnormalities in frontal and temporal cortical thickness and/or surface area. As a first step in achieving this aim we examined the differences in brain morphology between UHR participants with "absent to low" versus "moderate to high" degrees of childhood traumas, and between UHR individuals who transitioned (UHR-T) and individuals who did not transition (UHR-NT) to psychosis. We hypothesized that, where associations between any type of childhood trauma and 
transition are found, these would be mediated by reductions in frontal and/or temporal cortical thickness and/or surface area. We hypothesized that morphological aberrations may have the greatest mediating effect for sexual abuse, given that sexual abuse was shown to be a stronger risk factor for transition to psychosis than other forms of childhood trauma in our earlier study in the PACE 400 sample (Thompson et al., 2013).

\section{MATERIALS AND METHODS}

\subsection{Participants}

The participants included in the current study were part of the PACE 400 long-term followup study (for full details see (Nelson et al., 2013)). In brief, a total sample of 416 UHR individuals were recruited between 1993 and 2006 from the PACE (Personal Assessment and Clinical Evaluation) clinic, Orygen Youth Health (OYH), Melbourne, Australia, a specialist service for young people identified at UHR of psychosis. Individuals met the following criteria; aged 15-30 at the time of enrolment and fulfilling one or more of the following UHR group criteria: (1) attenuated psychotic symptoms (APS) experienced within the previous 12 months; (2) history of brief limited episode of full-blow psychotic psychopathology (BLIPS) which resolve within 7 days in the previous 12 months; (3) Trait vulnerability factors defined as either schizotypal personality disorder and/or family history of psychotic disorder in a first degree relative, plus decline in functioning or chronic low functioning. Exclusion criteria for PACE were presence of a current or past psychotic disorder, known organic cause for presentation, and past neuroleptic exposure equivalent to a total continuous haloperidol dose of $>15 \mathrm{mg}$. Exclusion criteria for brain imaging were neurological disorder, history of significant head injury, seizures or contraindication for magnetic resonance imaging (MRI). 
The local research and ethics committees (Melbourne Health) approved the study and all participants provided written informed consent.

Clinical and functional assessments including assessment of psychopathology, functioning, and structural magnetic resonance imaging (MRI) were conducted at baseline. Follow-up assessment included measures of psychopathology, functioning and past history of childhood trauma. Of the 416 participants in the PACE 400 study, 258 had baseline neuroimaging data and 233 had completed the childhood trauma questionnaire at follow-up; the number of participants with both MRI and trauma data was 88. Of those 88 participants, 21 were excluded due to data being acquired on a different (3T) machine, 1 was excluded due to organic psychoses and 4 were excluded due and poor MRI image quality (i.e., final current sample: $\mathrm{N}=62)$. Of the current sample, 24 (38.7\%) participants transitioned to psychosis during the follow-up period $(M$ years to follow-up=10.02, $\mathrm{SD}=1.52$; range=6.53 to 12.92 years). Participants included in the current study were older, more likely to have higher education, came from the earlier PACE cohorts, had a higher transition rate and lower baseline general psychopathology than those individuals in the overall cohort who did not have sufficient data for the current study $(N=353)$. They did not differ significantly in terms of UHR intake group, duration of untreated symptoms, severity of any type of childhood trauma, quality of life, or negative and depression symptoms (results can be provided upon request).

\subsection{Clinical and Functional Assessments}

Transition status information was derived from the Comprehensive Assessment of At Risk Mental States (CAARMS) (Yung et al., 2005) and public mental health records (see (Nelson 
et al., 2013) for further details). The Structured Clinical Interview for DSM-IV was used to determine specific psychotic (and other Axis I) disorder diagnoses at follow-up.

Other symptomatology was assessed using the Scale for the Assessment of Negative Symptoms (SANS (Andreasen, 1981)), the Brief Psychiatric Rating Scale (BPRS (Overall and Gorham, 1962)) and the Hamilton Rating Scale for Depression (HRSD (Hamilton, 1960)). Level of functioning was measured using the Global Assessment of functioning Scale (GAF (Endicott et al., 1976)). Quality of life was assessed using the Quality of Life Scale (QLS (Heinrichs et al., 1984)). Current IQ was assessed with the Wechsler Adult Intelligence Scale-Revised (WAIS-R (Wechsler, 1981)) or the Wechsler Abbreviated Scale of Intelligence (WASI (Wechsler, 1999)) following the procedure outlined in (Lin et al., 2011). Younger participants were assessed using the Wechsler Intelligence Scale for Children (WISC-III (Wechsler, 1991)). A sociodemographic interview was also performed at baseline.

\subsection{Trauma Measures}

History of childhood trauma was obtained at follow up using the brief (28-item) Childhood Trauma Questionnaire (CTQ) (Bernstein et al., 2003). This self-report questionnaire measures five dimensions of childhood trauma (i.e. occurring before the age of 18) through specific subscales: emotional, physical, and sexual abuse, and emotional and physical neglect. Each subscale consists of five items, scored on a 1-5 Likert scale (ranging from $1=$ "never true" to 5 = "very often true") with good internal consistency (Bernstein et al., 2003). We derived a total continuous CTQ (maltreatment) score, and continuous CTQ subscale scores for descriptive purposes. However, for the primary analyses we adopted a dichotomous approach and used previously defined cut-off scores for each subscale in order 
to identify two groups of individuals: those endorsing either zero-low levels of trauma (i.e. absent to low) or moderate-severe levels of trauma (i.e. moderate to high): $\geq 13$ for Emotional Abuse; $\geq 10$ for Physical Abuse; $\geq 8$ for Sexual Abuse; $\geq 15$ for Emotional Neglect; and $\geq$ 10 for Physical Neglect(Bernstein and Fink, 1998a, Bernstein and Fink, 1998b). We chose to adopt a categorical approach to examine CTQ data because the subscale scores of our sample were significantly skewed, as many participants reported no exposure to a given type of trauma.

\subsection{Brain Imaging}

\subsubsection{MRI acquisition and preprocessing}

Participants underwent magnetic resonance imaging (MRI) on a 1.5-T scanner (GE Signa; General Electric Medical Systems, Milwaukee, Wisconsin) at baseline. T-1 weighted images were obtained with the following parameters: 124 slices of $1.5 \mathrm{~mm}$ thickness, $\mathrm{TR}=1.43 \mathrm{~s}$, $\mathrm{TE}=3.3 \mathrm{~ms}$, flip angle $30^{\circ}$, matrix $256 \times 256$, and FOV $24 \mathrm{~cm}$. Images were pre-processed and surfaces were estimated using FreeSurfer v5.3 software (http://surfer.nmr.mgh.harvard.edu/)(Dale et al., 1999, Fischl et al., 2004). Processed images were visually inspected and any inaccuracies in cortical surface estimation were manually corrected by a rater who was blind to participant characteristics.

\subsubsection{MRI processing: Cortical thickness and surface area measures}

Based on previous psychosis transition literature (Bartholomeusz et al., 2016, Cannon et al., 2015, Edmiston et al., 2011, Jung et al., 2011, Kelly et al., 2013, Lim et al., 2018a, Sprooten et al., 2013, Sun et al., 2009b, Tognin et al., 2014, Ziermans et al., 2012), regions of interest (ROI/s) were selected and included prefrontal (including anterior cingulate) and temporal 
cortices defined using the Desikan-Killany Atlas (Desikan et al., 2006). Mean cortical thickness and total surface area values were estimated for each ROI using FreeSurfer. Mean cortical thickness and total surface area of the five prefrontal and two temporal ROI/s are presented in Figure 1 (and Supplementary Tables 1-10 for specific subdivisions). Data was exported to SPSS, version 22.0 (Chicago, Illinois) for statistical analyses.

\subsection{Statistical Analyses}

Normal distribution of quantitative variables was explored by means of the Shapiro-Wilk test. Paired student t-test, Pearson's chi-square or Fisher's Exact test was used to compare demographic variables between UHR-T and UHR-NT individuals.

\subsubsection{Group differences: Moderate to high vs absent to low trauma and UHR-T vs UHR-NT} groups

To explore the cortical morphological differences between "absent to low" and "moderate to high" trauma groups, Student $t$ test or Mann-Whitney U test were used according to the distribution of the variable. Student t test or Mann-Whitney U-tests were also performed to assess brain morphology in our ROI/s between UHR-T and UHR-NT groups. Cohen's $d$ or Rosenthal's $r$ were used to determine effect sizes across measurement indices according to the type of distribution. Significance was set at $\alpha=.05$. Adjustment for multiple comparisons was not made as planned comparisons were conducted based on a priori specified hypothesis (Freise, 2002, Gelman A, 2009, Perneger, 1998, Rothman, 1990, Saville, 1990).

\subsubsection{Mediation}

For any significant group differences in brain morphology (i.e. surface area, cortical 
thickness) that appeared both between childhood trauma groups (i.e., absent to low vs. moderate to high) and between UHR-NT and UHR-T individuals, mediation analyses were performed to assess whether cortical abnormalities mediated the relationship between trauma and transition (Cerin and Mackinnon, 2009). Note that, for mediation to occur it is not necessary to observe a significant direct effect of the independent variable on the dependent variable (Loeys et al., 2014). Mediation analyses were performed using a macro developed by Preacher and Hayes (Hayes, 2008). Mediation analysis allows for the examination of indirect pathways of influence from which $\mathrm{X}$ (childhood trauma) carries its effect on $\mathrm{Y}$ (transition). Mediation is indicated if the independent variable (X: childhood trauma) significantly predicts the mediator (M: brain morphology; the $a$ path), the mediator significantly predicts the dependent variable (Y: transition to psychosis) controlling for the independent variable (the $b$ path), and if the cross product of $a$ and $b$ is significant (Preacher and Hayes, 2004, 2008). The $a * b$ cross product tests the statistical significance of the difference between the total effect of the $\mathrm{X}$ on the $\mathrm{Y}$ ( $c$ path) and the direct effect ( $c^{\prime}$ path), which is the impact of the $\mathrm{X}$ on the $\mathrm{Y}$ adjusting for the effect of the mediator $(\mathrm{M})$. A bootstrapping method was applied to test the significance of the mediation effect (Hayes, 2008, Preacher and Hayes, 2008). This method generates bias corrected and accelerated confidence intervals (Preacher and Hayes, 2008). Significant mediation is indicated if the confidence intervals do not contain zero. For all analyses, 1000 resamples were taken and 95\% confidence intervals were used (Preacher and Hayes, 2008). Secondary mediation analyses were conducted to explore the potential effects of confounding variables. These variables were selected based on previously reported associations with childhood trauma, brain morphology or transition to psychosis (i.e. age, sex, medication status, psychopathology, IQ, quality of life, baseline functioning, duration of untreated symptoms 
prior to entry into the PACE clinic and year of entry into the PACE clinic) (Bechdolf et al., 2010, Nelson et al., 2013, Thompson et al., 2010, Thompson et al., 2013). The potential confounding variables that demonstrated significant associations with the primary variables of interest were entered as covariates and mediation analyses were re-run.

\section{RESULTS}

\subsection{Sociodemographic Characteristics and Reported Levels of Trauma}

UHR-T participants were not significantly different from UHR-NT participants in terms of age, gender, UHR intake group, length of follow-up, IQ, CTQ total score and the continuous variables for subscale scores (non-significant results were also found with CTQ dichotomous variables), year of entry into the clinic or severity of symptoms at study entry (see Table 1). There were significant between-group differences for functioning and duration of untreated symptoms with UHR-T presenting with longer duration of untreated symptoms, consistent with previous reports on this data set (Nelson et al., 2013, Thompson et al., 2013).

The mean total CTQ score for the whole UHR sample included in these analyses was 47.7 (range 25 to 94). The mean scores for the 5 subscales were similar to those scores reported previously (Thompson et al., 2013) - see Table 1. Females scored significantly higher than males on the CTQ sexual abuse subscale score $(\mathrm{t}=-2.222 \mathrm{p}=0.031)$, but not in any of the other CTQ subscales. In terms of the dichotomous CTQ variables, $18.0 \%$ of individuals scored in the moderate to high category for sexual abuse; $24.2 \%$ reported moderate to high levels of physical abuse; $45.2 \%$ reported moderate to high levels of emotional abuse; $33.9 \%$ reported moderate to high levels of emotional neglect and $22.6 \%$ of individuals scored in the moderate 
to high level on the physical neglect subscale. Participants in the moderate to high group of physical neglect were older (mean=22, $\mathrm{SD}=3.1$ ) than individuals in the absent to low group $($ mean=19.7 $\mathrm{SD}=3.7)(\mathrm{t}=-2.162 \mathrm{p}=0.035)$. We found no differences on the mean age of the individuals included in any of the other trauma subgroups.

\section{PLEASE INSERT TABLE 1 ABOUT HERE}

\subsection{Association Between Trauma, Brain Morphology and Transition to Psychosis}

Participants who reported moderate to high levels of childhood sexual abuse had significantly thinner cortices in the bilateral temporal (middle temporal gyrus - left: $t=2.218 \mathrm{p}=0.03$, $d=0.72$; right: $t=2.122 \mathrm{p}=0.04, d=0.70$ ) and right prefrontal lobe (superior frontal gyrus: $t=2.660 \mathrm{p}=0.01, d=0.79)$ relative to participants who reported absent to low levels of sexual abuse (see Supplementary Table 1 for comparison Student-t or Mann-Whitney U tests of all other ROI/s). In relation to surface area, individuals who reported moderate to high levels of childhood sexual abuse had significantly larger surface area in the caudal anterior division of the right cingulate $(\mathrm{U}=166.5 \mathrm{p}=0.04$, Rosenthal's $\mathrm{r}=0.26)$ compared to individuals who reported absent to low levels of childhood sexual abuse (see Supplementary Table 2). Participants reporting moderate to high levels of childhood physical abuse presented with increased cortical thickness in the right prefrontal lobe relative to participants reporting absent to low physical abuse (middle frontal gyrus rostral division: $\mathrm{U}=220 \mathrm{p}=0.03, r=0.28$; see Supplementary Table 3). No significant differences were found between participants reporting moderate to high and absent to low physical abuse in any of the surface area ROI measures (Supplementary Table 4). No differences were observed in ROI/s cortical thickness

or surface area, or transition status, between UHR individuals with moderate to high and 
absent to low emotional abuse, physical or emotional neglect (see Supplementary Tables 5 to 10$)$.

\section{PLEASE INSERT FIGURE 1 ABOUT HERE}

Participants who transitioned to psychosis had reduced cortical thickness in the right temporal lobe (middle temporal gyrus: $\mathrm{U}=319, \mathrm{p}=0.05, \mathrm{r}=-0.25$ ) and increased surface area in the right cingulate cortex (rostral anterior division: $\mathrm{U}=310, \mathrm{p}=0.04, \mathrm{r}=0.27$ ).

\subsection{Mediation effects of Brain Morphology on Transition to Psychosis}

Given that only cortical thickness in the right middle temporal gyrus significantly differed both between childhood trauma (i.e., moderate to high vs. absent to low degree of sexual trauma) and between UHR-NT and UHR-T groups, mediation analysis was used to test whether decreased cortical thickness in this ROI mediated the association between presence of childhood sexual abuse and transition to psychosis. Our primary model was built to explore the relationship between our variables of interest. Possible confounding variables were checked in a secondary model, which included those variables that demonstrated significant associations with the primary variables of interest (i.e. physical and emotional abuse, physical and emotional neglect, baseline functioning, duration of untreated symptoms and sex -results can be provided upon request). A final secondary mediation model was re-run including baseline functioning and duration of untreated symptoms as covariates, as these were found to have significant partial effects on the dependent variable in the model (i.e. transition). Although presence of physical abuse was also associated with variables of interest, given the inherent nature of overlap in the incidence of sexual and physical abuse and the likelihood 
that controlling for physical abuse may be masking a potentially important real-world association, our final secondary model did not control for physical abuse.

In the primary model, bias corrected $95 \%$ confidence interval indicated that there was a significant mediation effect ( $a^{*} b$ path) (CI .001, 1.65) (see Figure 2). The model explained $9.2 \%$ of the variance of transition to psychosis.

\section{INSERT FIGURE 2 ABOUT HERE}

In the secondary analysis (controlling for confounding covariates), partial effects of control variables on transition were significant for baseline functioning $($ Coeff $=-0.09 \mathrm{p}<0.01)$ and

duration of untreated symptoms $($ Coeff $=0.002 \mathrm{p}=0.02)$. The mediation effect $(a * b$ path $)$ of the right middle temporal gyrus was not significant (bias corrected 95\% CI -.13, 1.60). The model explained $40.2 \%$ of the variance of transition to psychosis.

\section{DISCUSSION}

This is the first study to examine the relationship between various types of childhood trauma and potential morphological brain risk markers with transition to psychosis in a UHR cohort. Significant cortical thickness reductions in prefrontal (right superior frontal gyrus) and temporal (bilateral middle temporal gyrus) brain regions were observed in individuals at UHR for psychosis who reported moderate to high levels of childhood sexual abuse when compared to those who reported absent to low levels of childhood sexual abuse. In contrast, participants who experienced moderate to high levels of childhood physical abuse were found to have increased cortical thickness in a distinct prefrontal structure (right middle frontal 
gyrus). No significant differences in brain measures were detected for emotional abuse, physical or emotional neglect. These data suggest that morphology of particularly superior prefrontal and temporal structures may be vulnerable to the stress of sexual and physical abuse, but not other forms of trauma. Furthermore, UHR individuals who transitioned to psychosis presented with reduced cortical thickness in the right middle temporal gyrus. Mediation analyses found that history of childhood sexual trauma had a significant indirect effect on transition via cortical thickness of the right middle temporal gyrus, which acted as a significant mediator. There were no direct associations between any type of trauma investigated and transition to psychosis. Therefore, our results suggest that childhood sexual abuse negatively impacted on cortical development of the right temporal gyrus, and that this in turn heightened the risk of transition to psychosis in our sample.

Our neuroimaging results are partially in line with previous trauma literature, where decreased cortical thickness in the right frontal lobe, including the superior frontal gyrus, has been observed in children and youth who have a history of maltreatment (Kelly et al., 2013, Lim et al., 2018a). Kelly and colleagues (2013) additionally found reduced surface area in the left middle temporal cortex of maltreated children, whereas the current study, and the study by Lim and colleagues (2018), did not find any relationship between childhood trauma and cortical surface area. Discrepancies between these two studies may be due to the definition of childhood trauma (i.e. exposure to any type of trauma rated by a social worker for (Kelly et al., 2013) and physical abuse only for (Lim et al., 2018a) ) or the samples being at differing neurodevelopmental stages (Mean age; 12.3 for (Kelly et al., 2013) and mean age. 17.6 for (Lim et al., 2018a)). Contrary to previous research, the current study failed to 
find associations between childhood traumas and several ROIs, including those in the left prefrontal lobe and bilateral cingulate, where primarily cortical volume reductions of these regions have been previously associated with trauma histories (Andersen et al., 2008, Kelly et al., 2013, Lim et al., 2018a). Our findings further add to the mixed trauma literature (McCrory et al., 2011), and inconsistencies are likely influenced by the differences in age and developmental stage of the samples, the heterogeneity in psychiatric presentations of individuals within and between samples, and various demographic and methodological factors.

Our neuroimaging results are partially in line with the psychosis-trauma literature, where severity of sexual abuse has been found associated with grey matter volume loss in the prefrontal cortex in individuals with psychosis (Sheffield et al., 2013). Habets and colleagues (Habets et al., 2011) found that exposure to trauma in childhood was associated with cerebral cortical thinning in individuals with schizophrenia (Habets et al., 2011). The opposite relationship was found in genetic at-risk siblings of patients with schizophrenia, for which higher levels of trauma were associated with increased whole brain cortical thickness (Habets et al., 2011). This was as study using a general measure of developmental trauma based on the mean of the items of a short form of the CTQ assessing the five types of trauma; emotional, physical, sexual abuse and emotional and physical neglect. We observed a similar increase in cortical thickness of the right middle frontal gyrus in UHR participants who had been exposed to high levels of childhood physical abuse. Given there is some evidence that sexual trauma may have a higher load than other forms of trauma for psychosis risk (Bechdolf et al., 2010, Kraan et al., 2015, Thompson et al., 2013), and that sexual and physical trauma typically occur contemporaneously, it is difficult to explain why such an increase would be 
associated with only physical trauma. However, drawing on the broader trauma literature, a recent study on youth exposed to physical childhood abuse showed increased cortical volume in the left inferior and middle temporal gyri relative to controls (Lim et al., 2018a). This study excluded sexual abuse because of its differential effects on brain structure. Different effects of trauma subtypes on brain structure and function have also been reported by a recent review by (Cassiers et al., 2018). However none of the trauma subtypes examined in this review (i.e. sexual, emotional and physical abuse and neglect) were associated with evidence of increased structural or functional integrity. Our findings further suggest that the increase in cortical thickness of the right middle frontal gyrus does not impact on the later development of psychosis, given no association was found with transition. It is possible that increases in frontal cortical thickness confer risk for development of other psychiatric illnesses, or poor functional outcomes, which were not the focus of the current study. Alternatively, increased cortical thickness in the middle frontal gyrus could be reflective of some form of resilience, adaptation or a neuroprotective mechanism that may moderate the outcome of childhood physical abuse, for example via dysregulation of hypothalamic pituitary adrenal (HPA)-axis functioning (Frodl and O'Keane, 2013, McCrory et al., 2011). It may be that the cascade of neurobiochemical events which result from HPA-axis dysregulation post exposure to a stressor (such as childhood abuse) contributes to brain abnormalities that are either protective or detrimental, depending on other predisposing and precipitating factors; abnormalities are often evident during the premorbid and early stages of psychosis (Cotter and Pariante, 2002, Hart and Rubia, 2012, McEwen and Magarinos, 2001, Selemon and Goldman-Rakic, 1999). However this is highly speculative and further research is needed to define the consequences of HPA axis dysregulation. While developmental changes and sex differences could be playing a role, there is currently not 
enough literature to draw strong conclusions based on patterns of results. Thus, future research should examine the inter-relationships between cortical thickness, different types of childhood trauma, sex, developmental changes, HPA-axis functioning and transition to psychosis and other psychiatric disorders.

Some of the brain regions that demonstrated structural brain differences in UHR have also been implicated in stress exposure. In our sample, UHR-T individuals presented with a specific decrease of cortical thickness in the middle temporal gyrus and increase of surface area in the cingulate cortex relative to UHR-NT individuals, regions previously and repeatedly found to be aberrant in UHR-T (Cannon et al., 2015, Jung et al., 2011, Ziermans et al., 2012). Despite this, we failed to find an association between many of our ROIs and transition; it may be that cortical thickness in these regions have their effect 'earlier in the chain', by for example, producing attenuated psychotic symptoms (or even general psychopathology), and therefore contribute less to the evolution of a UHR individual transitioning into full-threshold psychosis. We additionally found decreased cortical thickness in the right middle temporal gyrus to act as a significant mediator for the effects childhood sexual trauma had on transition, where a high degree of trauma led to decreased thickness, and in turn higher risk of transition to psychosis. As expected, history of childhood sexual trauma had a stronger effect than other forms of trauma, being the only trauma subtype to (indirectly) effect transition. However, mediation was no longer significant when we accounted for the effects of global functioning and duration of untreated symptoms, nor did we find any other ROI to act as a mediator. In this regard, while childhood sexual trauma appears to be an important contributing factor for risk of psychosis and may exert its effects by influencing cortical thickness development of the right middle temporal gyrus, it is likely 
to work in concert with other known (or unknown) moderating and/or mediating factors (Myin-Germeys and van Os, 2007). For example, it has been suggested that the effects of maltreatment may be moderated by genotype, where epigenetic research has linked certain polymorphisms to resilience and vulnerability for exposure to childhood trauma and later development of psychopathology (McCrory et al., 2011).

Limitations of this study include the relatively small sample size, which might lead to unrepresentative childhood trauma exposure and transition to psychosis subgroups (i.e. transition rate was higher compared to the main study from which this sample was derived: $39.7 \%$ vs. $22.8 \%$ (Thompson et al., 2013)). While we found no direct association between childhood sexual abuse and transition in this sample, mediation is still statistically and theoretically possible (Loeys et al., 2014). Lack of a direct association may be attributable to the fact that there are likely many mediating pathways linking sexual trauma and transition; if these pathways differ in the valence of their direction, they may cancel each other out (Cerin and Mackinnon, 2009). However, it is also possible that lack of a direct effect might be due to the sample being underpowered, as the previous study of Thompson and colleagues ${ }^{4}$ (from which the current sample was derived), did find a direct effect with a larger sample ( $N=233$ ). Childhood trauma measures were based on self-report and obtained at the followup assessment (i.e., on average 10.12 years post-baseline assessment); recall bias and subjective perception of the exposure to childhood trauma might thus be an inherent limitation of the study design. Even though studies have suggested that trauma reports are consistent regardless of the time when they are reported, and the CTQ has shown good convergence with other structured interview-rated measures of trauma (Bernstein et al., 1994, Fisher et al., 2011), findings may have been different had the we collected collateral 
information. Childhood trauma subscales are also likely to overlap, as individuals that have suffered from one type of abuse/neglect might also have suffered from other types of abuse/neglect. We did not correct for multiple comparisons in the group analyses, which might be perceived as a limitation. However, there is no current agreement among statisticians about the need to make p-value adjustments while analyzing data (Althouse, 2016, Armstrong, 2014, Freise, 2002, Perneger, 1998, Rothman, 1990) We choose to report all comparisons, p-values and effect sizes and regard our findings as tentative (Althouse, 2016, Rothman et al., 2012). Multiple testing correction via false discovery rate (FDR) estimation method (FDR of 20\%; q=0.20)(Benjamini and Yekutieli, 2001) would have resulted only in significant findings for the differences in cortical thickness in the temporal (right middle temporal gyrus) and prefrontal lobe (right superior frontal gyrus) between UHR participants with absent to low and moderate to high childhood sexual abuse; and for differences in the temporal (right middle temporal gyrus) and prefrontal lobe (right middle frontal caudal gyrus) between UHR participants who transitioned to a psychosis diagnosis and those who did not transitioned. Females were over-represented in the moderate to high sexual abuse group. The proportion of females was higher than males in the moderate to high sexual abuse subgroup $(\mathrm{X} 2=6.17 \mathrm{p}=0.01)$, but not in any of the other trauma subgroups. Lastly, the current study focused on a small number of ROIs, thus a whole-brain interdisciplinary approach that incorporates environmental, genetic, biological and behavioural measures should be adopted for future research in order to better understand the complex interplay between the breadth of psychosis risk factors and illness outcomes. Similarly, various types of childhood trauma can have effects on a wide array of mental health outcomes (Caspi et al., 2014), thus the neurobiological sequelae of childhood trauma is likely relevant to outcomes other than transition to psychosis, such as functioning and other 
mental disorders; the specificity of the present findings to psychosis transition thus warrants further investigation.

In summary, our results provide evidence for the impact of childhood sexual trauma on decreased cortical thickness and for its potential predictive value for transition to psychosis, highlighting the need to target various types of childhood trauma in both assessment and treatment of individuals at UHR for psychosis. Treatment approaches to improve functioning and symptoms that take into account trauma history (particularly sexual trauma) might be useful to mitigate both its biological and psychosocial effects in this population (van den Berg et al., 2015, van den Berg et al., 2016). Further longitudinal studies are needed to precisely understand this relationship and the specific role of various types of childhood trauma and cortical aberrations as plausible mechanisms that may contribute to the onset of psychosis. 


\section{AUTHOR DISCLOSURE}

\section{Financial support}

MR-C was supported by a Ramon y Cajal Research Fellowship (RYC-2017-23144), Spanish Ministry of Science, Innovation and Universities, a Sara Borrell Health Research Fellowship from the Institute of Health Carlos III, Spanish Ministry of Economy and Competitiveness, an Alicia Koplowitz Research Grant and Short-Term Visiting Fellowship from the Alicia Koplowitz Foundation, a Fellowship Award from the Health Research Institute, Hospital Gregorio Marañon (Madrid, Spain), and a NARSAD independent investigator grant (24628) from the Brain \& Behavior Research Foundation. MR-C was partially supported by the Spanish Ministry of Economy and Competitiveness, Instituto de Salud Carlos III (PI15/00723, PI18/00753), co-financed by ERDF Funds from the European Commission, “A way of making Europe”, CIBERSAM, Madrid Regional Government (B2017/BMD-3740 AGES-CM-2), European Union Structural Funds and European Union Seventh Framework Program, European Union H2020 Program under the Innovative Medicines Initiative 2 Joint; Fundación Familia Alonso, Fundación Alicia Koplowitz and Fundación Mutua Madrileña. BN was supported by an NHMRC Senior Research Fellowship (1137687). AL is supported by a NHMRC Career Development Fellowship (1148793). SW was supported by a NHMRC Career Development Fellowship (1125504). CP has received a NHMRC Australian-based Senior Principal Research Fellowship (628386), NARSAD Distinguished Investigator Award, and NHMRC Program Grant (566529); he has received grant support from JanssenCilag, Eli Lilly, Hospira (Mayne), Astra Zeneca and has provided consultancy to JanssenCilag, Eli Lilly, Hospira (Mayne), Astra Zeneca, Pfizer, Schering Plough, Lundbeck. He has undertaken investigator initiated studies supported by Eli Lilly, Hospira, Janssen Cilag and Astra Zeneca. 


\section{Contributors}

Authors MR-C, SW, BN, AT, CP and CB designed the study. Author MR-C managed the literature searches and wrote the protocol. Authors MR-C, SW, CB managed the analyses. Authors MR-C and EG undertook the brain imaging analyses. Authors MR-C and SW undertook the statistical analysis and author MR-C wrote the first draft of the manuscript. All authors contributed to and have approved the final manuscript.

Conflicts of interest: The authors have no conflicts of interest.

Ethical standards: The authors assert that all procedures contributing to this work comply with the ethical standards of the relevant national and institutional committees on human experimentation and with the Helsinki Declaration of 1975, as revised in 2008.

\section{Acknowledgements}

We thank Dr. Yasser Aleman for his assistance with the figures design, Dr. Joost Janssen for independently reviewing the manuscript and Dr. Jose de Arriba who assisted with the preparation of the manuscript. 


\section{REFERENCES}

Althouse, A. D. (2016). Adjust for Multiple Comparisons? It's Not That Simple. The statistician's Page. Ann Thorac Surg 101.

Andersen, S. L., Tomada, A., Vincow, E. S., Valente, E., Polcari, A. \& Teicher, M. H. (2008). Preliminary evidence for sensitive periods in the effect of childhood sexual abuse on regional brain development. J Neuropsychiatry Clin Neurosci 20, 292-301.

Andreasen, N. C. (1981). Scale for the Assessment of Negative Symptoms (SANS).

Armstrong, R. A. (2014). When to use the Bonferroni correction. Ophthalmic Physiol. Opt. 34, 502-508.

Arseneault, L., Cannon, M., Fisher, H. L., Polanczyk, G., Moffitt, T. E. \& Caspi, A. (2011). Childhood trauma and children's emerging psychotic symptoms: a genetically sensitive longitudinal cohort study. American Journal of Psychiatry 168, 65-72.

Bartholomeusz, C. F., Cropley, V. L., Wannan, C., Di Biase, M., McGorry, P. D. \& Pantelis, C. (2016). Structural neuroimaging across early-stage psychosis: Aberrations in neurobiological trajectories and implications for the staging model. Aust N Z J Psychiatry.

Bartholomeusz, C. F., Cropley, V. L., Wannan, C., Di Biase, M., McGorry, P. D. \& Pantelis, C. (2017). Structural neuroimaging across early-stage psychosis: Aberrations in neurobiological trajectories and implications for the staging model. Australian \& New Zealand Journal of Psychiatry 51, 455-476.

Bechdolf, A., Thompson, A., Nelson, B., Cotton, S., Simmons, M. B., Amminger, G. P., Leicester, S., Francey, S. M., McNab, C., Krstev, H., Sidis, A., McGorry, P. D. \& Yung, A. R. (2010). Experience of trauma and conversion to psychosis in an ultra-high-risk (prodromal) group. Acta Psychiatrica Scandinavica 121, 377-384.

Bendall, S., Jackson, H. J., Hulbert, C. A. \& McGorry, P. D. (2008). Childhood trauma and psychotic disorders: a systematic, critical review of the evidence. Schizophrenia Bulletin 34, 568-79.

Benjamini, Y. \& Yekutieli, D. (2001). The control of the false discovery rate in multiple testing under dependency. The annals of statistics 29, 1165-1188.

Bernstein, D. \& Fink, L. (1998a). Manual for the childhood trauma questionnaire. The Psychological Corporation, New York.

Bernstein, D. P. \& Fink, L. (1998b). Childhood trauma questionnaire: A retrospective self-report: Manual. Psychological Corporation.

Bernstein, D. P., Fink, L., Handelsman, L., Foote, J., Lovejoy, M., Wenzel, K., Sapareto, E. \& Ruggiero, J. (1994). Initial reliability and validity of a new retrospective measure of child abuse and neglect. Am J Psychiatry 151, 1132-6.

Bernstein, D. P., Stein, J. A., Newcomb, M. D., Walker, E., Pogge, D., Ahluvalia, T., Stokes, J., Handelsman, L., Medrano, M. \& Desmond, D. (2003). Development and validation of a brief screening version of the Childhood Trauma Questionnaire. Child abuse \& neglect 27, 169-190.

Blakemore, S. J. (2012). Imaging brain development: the adolescent brain. Neuroimage 61, 397-406.

Brown, T. T. \& Jernigan, T. L. (2012). Brain development during the preschool years. Neuropsychol Rev 22, 313-33.

Cannon, T. D., Chung, Y., He, G., Sun, D., Jacobson, A., van Erp, T. G., McEwen, S., Addington, J., Bearden, C. E., Cadenhead, K., Cornblatt, B., Mathalon, D. H., McGlashan, T., Perkins, D., Jeffries, C., Seidman, L. J., Tsuang, M., Walker, E., Woods, S. W. \& Heinssen, R. (2015). Progressive reduction in cortical thickness as psychosis develops: a multisite longitudinal neuroimaging study of youth at elevated clinical risk. Biol Psychiatry 77, 147-57. 
Caspi, A., Houts, R. M., Belsky, D. W., Goldman-Mellor, S. J., Harrington, H., Israel, S., Meier, M. H., Ramrakha, S., Shalev, I., Poulton, R. \& Moffitt, T. E. (2014). The p Factor: One General Psychopathology Factor in the Structure of Psychiatric Disorders? Clin Psychol Sci 2, 119-137. Cassiers, L. L. M., Sabbe, B. G., Schmaal, L., Veltman, D. J., Penninx, B. W. \& Van Den Eede, F. (2018). Structural and functional brain abnormalities associated with exposure to different childhood trauma subtypes: A systematic review of neuroimaging findings. Frontiers in psychiatry 9, 329.

Cerin, E. \& Mackinnon, D. P. (2009). A commentary on current practice in mediating variable analyses in behavioural nutrition and physical activity. Public Health Nutr 12, 1182-8.

Cotter, D. \& Pariante, C. M. (2002). Stress and the progression of the developmental hypothesis of schizophrenia. Br J Psychiatry 181, 363-5.

Croft, J., Heron, J., Teufel, C., Cannon, M., Wolke, D., Thompson, A., Houtepen, L. \& Zammit, S. (2018). Association of Trauma Type, Age of Exposure, and Frequency in Childhood and Adolescence With Psychotic Experiences in Early Adulthood. JAMA Psychiatry.

Cunningham, T., Hoy, K. \& Shannon, C. (2016). Does childhood bullying lead to the development of psychotic symptoms? A meta-analysis and review of prospective studies. Psychosis 8, 48-59.

Cutajar, M. C., Mullen, P. E., Ogloff, J. R., Thomas, S. D., Wells, D. L. \& Spataro, J. (2010). Schizophrenia and other psychotic disorders in a cohort of sexually abused children. Arch Gen Psychiatry 67, 1114-9.

Dale, A. M., Fischl, B. \& Sereno, M. I. (1999). Cortical surface-based analysis. I. Segmentation and surface reconstruction. Neuroimage $\mathbf{9}, 179-94$.

Desikan, R. S., Ségonne, F., Fischl, B., Quinn, B. T., Dickerson, B. C., Blacker, D., Buckner, R. L., Dale, A. M., Maguire, R. P. \& Hyman, B. T. (2006). An automated labeling system for subdividing the human cerebral cortex on MRI scans into gyral based regions of interest. Neuroimage 31, 968-980.

Edmiston, E. E., Wang, F., Mazure, C. M., Guiney, J., Sinha, R., Mayes, L. C. \& Blumberg, H. P. (2011). Corticostriatal-limbic gray matter morphology in adolescents with self-reported exposure to childhood maltreatment. Arch Pediatr Adolesc Med 165, 1069-77.

Endicott, J., Spitzer, R. L., Fleiss, J. L. \& Cohen, J. (1976). The global assessment scale. A procedure for measuring overall severity of psychiatric disturbance. Arch Gen Psychiatry 33, 766-71.

Fischl, B., van der Kouwe, A., Destrieux, C., Halgren, E., Segonne, F., Salat, D. H., Busa, E., Seidman, L. J., Goldstein, J., Kennedy, D., Caviness, V., Makris, N., Rosen, B. \& Dale, A. M. (2004). Automatically parcellating the human cerebral cortex. Cereb Cortex 14, 11-22.

Fisher, H. L., Craig, T. K., Fearon, P., Morgan, K., Dazzan, P., Lappin, J., Hutchinson, G., Doody, G. A., Jones, P. B., McGuffin, P., Murray, R. M., Leff, J. \& Morgan, C. (2011). Reliability and comparability of psychosis patients' retrospective reports of childhood abuse. Schizophr Bull 37, 546-53.

Freise, R. J. (2002). Do multiple outcome measures require p-value adjustment? BMC Medical Research Methodology 2.

Frodl, T. \& O'Keane, V. (2013). How does the brain deal with cumulative stress? A review with focus on developmental stress, HPA axis function and hippocampal structure in humans. Neurobiol Dis 52, 24-37.

Gelman A, H. J., Yajima M (2009). Why we (usually) don't have to worry about multiple comparisons. (https://arxiv. org/abs/0907.2478).

Habets, P., Marcelis, M., Gronenschild, E., Drukker, M. \& van Os, J. (2011). Reduced cortical thickness as an outcome of differential sensitivity to environmental risks in schizophrenia. Biol Psychiatry 69, 487-94. 
Hamilton, M. (1960). A rating scale for depression. J Neurol Neurosurg Psychiatry 23, 56-62. Hart, H. \& Rubia, K. (2012). Neuroimaging of child abuse: a critical review. Frontiers in Human Neuroscience 6, 52.

Hayes, P. a. (2008). SPSS Macro for Multiple Mediation Written by Andrew F. Hayes, The Ohio State University. http://www.afhayes.com.

Heinrichs, D. W., Hanlon, T. E. \& Carpenter, W. T. (1984). The Quality of Life Scale: an instrument for rating the schizophrenic deficit syndrome. Schizophrenia bulletin 10, 388.

Isvoranu, A. M., van Borkulo, C. D., Boyette, L. L., Wigman, J. T., Vinkers, C. H. \& Borsboom, D. (2017). A Network Approach to Psychosis: Pathways Between Childhood Trauma and Psychotic Symptoms. Schizophr Bull 43, 187-196.

Jung, W. H., Kim, J. S., Jang, J. H., Choi, J. S., Jung, M. H., Park, J. Y., Han, J. Y., Choi, C. H., Kang, D. H., Chung, C. K. \& Kwon, J. S. (2011). Cortical thickness reduction in individuals at ultrahigh-risk for psychosis. Schizophr Bull 37, 839-49.

Kelly, P. A., Viding, E., Wallace, G. L., Schaer, M., De Brito, S. A., Robustelli, B. \& McCrory, E. J. (2013). Cortical thickness, surface area, and gyrification abnormalities in children exposed to maltreatment: neural markers of vulnerability? Biol Psychiatry 74, 845-52.

Kraan, T., Velthorst, E., Smit, F., de Haan, L. \& van der Gaag, M. (2015). Trauma and recent life events in individuals at ultra high risk for psychosis: review and meta-analysis. Schizophr Res 161, 143-9.

Kraan, T. C., Velthorst, E., Themmen, M., Valmaggia, L., Kempton, M. J., McGuire, P., van Os, J., Rutten, B. P. F., Smit, F., de Haan, L. \& van der Gaag, M. (2018). Child Maltreatment and Clinical Outcome in Individuals at Ultra-High Risk for Psychosis in the EU-GEI High Risk Study. Schizophr Bull 44, 584-592.

Lim, L., Hart, H., Mehta, M., Worker, A., Simmons, A., Mirza, K. \& Rubia, K. (2018a). Grey matter volume and thickness abnormalities in young people with a history of childhood abuse. Psychol Med 48, 1034-1046.

Lim, L., Hart, H., Mehta, M., Worker, A., Simmons, A., Mirza, K. \& Rubia, K. (2018b). Grey matter volume and thickness abnormalities in young people with a history of childhood abuse. Psychological medicine 48, 1034-1046.

Lim, L., Radua, J. \& Rubia, K. (2014). Gray matter abnormalities in childhood maltreatment: a voxel-wise meta-analysis. Am J Psychiatry 171, 854-63.

Lin, A., Wood, S. J., Nelson, B., Brewer, W. J., Spiliotacopoulos, D., Bruxner, A., Broussard, C., Pantelis, C. \& Yung, A. R. (2011). Neurocognitive predictors of functional outcome two to 13years after identification as ultra-high risk for psychosis. Schizophrenia Research 132, 1-7.

Liu, C. H., Keshavan, M. S., Tronick, E. \& Seidman, L. J. (2015). Perinatal risks and childhood premorbid indicators of later psychosis: next steps for early psychosocial interventions. Schizophrenia bulletin 41, 801-816.

Loeys, T., Moerkerke, B. \& Vansteelandt, S. (2014). A cautionary note on the power of the test for the indirect effect in mediation analysis. Front Psychol 5, 1549.

McCrory, E., De Brito, S. A. \& Viding, E. (2011). The impact of childhood maltreatment: a review of neurobiological and genetic factors. Front Psychiatry 2, 48.

McEwen, B. S. \& Magarinos, A. M. (2001). Stress and hippocampal plasticity: implications for the pathophysiology of affective disorders. Human Psychopharmacology: Clinical and Experimental 16, S7-S19.

McGrath, J. J., McLaughlin, K. A., Saha, S., Aguilar-Gaxiola, S., Al-Hamzawi, A., Alonso, J., Bruffaerts, R., de Girolamo, G., de Jonge, P., Esan, O., Florescu, S., Gureje, O., Haro, J. M., Hu, C., Karam, E. G., Kovess-Masfety, V., Lee, S., Lepine, J. P., Lim, C. C., Medina-Mora, M. E., Mneimneh, Z., Pennell, B. E., Piazza, M., Posada-Villa, J., Sampson, N., Viana, M. C., Xavier, M., Bromet, E. J., Kendler, K. S. \& Kessler, R. C. (2017). The association between childhood 
adversities and subsequent first onset of psychotic experiences: a cross-national analysis of 23 998 respondents from 17 countries. Psychol Med 47, 1230-1245.

McIntosh, A. M., Owens, D. C., Moorhead, W. J., Whalley, H. C., Stanfield, A. C., Hall, J., Johnstone, E. C. \& Lawrie, S. M. (2011). Longitudinal volume reductions in people at high genetic risk of schizophrenia as they develop psychosis. Biol Psychiatry 69, 953-8.

Mechelli, A., Riecher-Rossler, A., Meisenzahl, E. M., Tognin, S., Wood, S. J., Borgwardt, S. J., Koutsouleris, N., Yung, A. R., Stone, J. M., Phillips, L. J., McGorry, P. D., Valli, I., Velakoulis, D., Woolley, J., Pantelis, C. \& McGuire, P. (2011). Neuroanatomical abnormalities that predate the onset of psychosis: a multicenter study. Arch Gen Psychiatry 68, 489-95.

Mirsky, A. F. \& Duncan, C. C. (1986). Etiology and expression of schizophrenia: neurobiological and psychosocial factors. Annu Rev Psychol 37, 291-319.

Myin-Germeys, I. \& van Os, J. (2007). Stress-reactivity in psychosis: evidence for an affective pathway to psychosis. Clinical psychology review 27, 409-424.

Nelson, B., Yuen, H. P., Wood, S. J., Lin, A., Spiliotacopoulos, D., Bruxner, A., Broussard, C., Simmons, M., Foley, D. L., Brewer, W. J., Francey, S. M., Amminger, G. P., Thompson, A., McGorry, P. D. \& Yung, A. R. (2013). Long-term Follow-up of a Group at Ultra High Risk ("Prodromal") for Psychosis: The PACE 400 Study. JAMA Psychiatry, 1-10.

Niendam, T. A., Jalbrzikowski, M. \& Bearden, C. E. (2009). Exploring predictors of outcome in the psychosis prodrome: implications for early identification and intervention. Neuropsychol Rev 19, 280-93.

Overall, J. E. \& Gorham, D. R. (1962). THE BRIEF PSYCHIATRIC RATING SCALE. Psychological Reports 10, 799-812.

Pantelis, C., Velakoulis, D., McGorry, P. D., Wood, S. J., Suckling, J., Phillips, L. J., Yung, A. R., Bullmore, E. T., Brewer, W., Soulsby, B., Desmond, P. \& McGuire, P. K. (2003). Neuroanatomical abnormalities before and after onset of psychosis: a cross-sectional and longitudinal MRI comparison. Lancet 361, 281-288.

Perneger, T. V. (1998). What's wrong with Bonferroni adjustments. BMJ 316, 1236-1238.

Preacher, K. J. \& Hayes, A. F. (2004). SPSS and SAS procedures for estimating indirect effects in simple mediation models. Behavior research methods, instruments, \& computers 36, 717-731. Preacher, K. J. \& Hayes, A. F. (2008). Asymptotic and resampling strategies for assessing and comparing indirect effects in multiple mediator models. Behavior research methods 40, 879891.

Rothman, K., Greenland, S. \& Lash, T. (2012). Modern epidemiology third, mid-cycle revision edition. Philadelphia: Lippincott, Williams and Wilkins.

Rothman, K. J. (1990). No adjustments are needed for multiple comparisons. Epidemiology 1, 43-46.

Saville, D. J. (1990). Multiple Comparison Procedures: The Practical Solution.. The American Statistician 44, 174-180.

Selemon, L. D. \& Goldman-Rakic, P. S. (1999). The reduced neuropil hypothesis: a circuit based model of schizophrenia. Biol Psychiatry 45, 17-25.

Sheffield, J. M., Williams, L. E., Woodward, N. D. \& Heckers, S. (2013). Reduced gray matter volume in psychotic disorder patients with a history of childhood sexual abuse. Schizophr Res 143, 185-91.

Sowell, E. R., Thompson, P. M., Holmes, C. J., Batth, R., Jernigan, T. L. \& Toga, A. W. (1999). Localizing age-related changes in brain structure between childhood and adolescence using statistical parametric mapping. Neuroimage 9, 587-97.

Spauwen, J., Krabbendam, L., Lieb, R., Wittchen, H. U. \& van Os, J. (2006). Impact of psychological trauma on the development of psychotic symptoms: relationship with psychosis proneness. Br J Psychiatry 188, 527-33. 
Sprooten, E., Papmeyer, M., Smyth, A. M., Vincenz, D., Honold, S., Conlon, G. A., Moorhead, T. W., Job, D., Whalley, H. C., Hall, J., McIntosh, A. M., Owens, D. C., Johnstone, E. C. \& Lawrie, S. M. (2013). Cortical thickness in first-episode schizophrenia patients and individuals at high familial risk: a cross-sectional comparison. Schizophr Res 151, 259-64.

Sun, D., Phillips, L., Velakoulis, D., Yung, A., McGorry, P. D., Wood, S. J., van Erp, T. G., Thompson, P. M., Toga, A. W., Cannon, T. D. \& Pantelis, C. (2009a). Progressive brain structural changes mapped as psychosis develops in 'at risk' individuals. Schizophrenia Research 108, 85-92.

Sun, D., Phillips, L., Velakoulis, D., Yung, A., McGorry, P. D., Wood, S. J., van Erp, T. G., Thompson, P. M., Toga, A. W., Cannon, T. D. \& Pantelis, C. (2009b). Progressive brain structural changes mapped as psychosis develops in 'at risk' individuals. Schizophr Res 108, 8592.

Sun, D., Stuart, G. W., Jenkinson, M., Wood, S. J., McGorry, P. D., Velakoulis, D., van Erp, T. G., Thompson, P. M., Toga, A. W., Smith, D. J., Cannon, T. D. \& Pantelis, C. (2009c). Brain surface contraction mapped in first-episode schizophrenia: a longitudinal magnetic resonance imaging study. Molecular Psychiatry 14, 976-86.

Tamnes, C. K., Herting, M. M., Goddings, A. L., Meuwese, R., Blakemore, S. J. \& Dahl, R. E. (2017). Development of the Cerebral Cortex across Adolescence: A Multisample Study of InterRelated Longitudinal Changes in Cortical Volume, Surface Area, and Thickness. 37, 3402-3412. Teicher, M. H., Andersen, S. L., Polcari, A., Anderson, C. M. \& Navalta, C. P. (2002). Developmental neurobiology of childhood stress and trauma. Psychiatr Clin North Am 25, 397426, vii-viii.

Teicher, M. H., Anderson, C. M. \& Polcari, A. (2012). Childhood maltreatment is associated with reduced volume in the hippocampal subfields CA3, dentate gyrus, and subiculum. Proc Natl Acad Sci U S A 109, E563-72.

Thompson, A., Nelson, B., McNab, C., Simmons, M., Leicester, S., McGorry, P. D., Bechdolf, A. \& Yung, A. R. (2010). Psychotic symptoms with sexual content in the "ultra high risk" for psychosis population: frequency and association with sexual trauma. Psychiatry Research 177, 84-91.

Thompson, A. D., Nelson, B., Yuen, H. P., Lin, A., Amminger, G. P., McGorry, P. D., Wood, S. J. \& Yung, A. R. (2013). Sexual Trauma Increases the Risk of Developing Psychosis in an Ultra High-Risk "Prodromal" Population. Schizophr Bull.

Tognin, S., Riecher-Rossler, A., Meisenzahl, E. M., Wood, S. J., Hutton, C., Borgwardt, S. J., Koutsouleris, N., Yung, A. R., Allen, P., Phillips, L. J., McGorry, P. D., Valli, I., Velakoulis, D., Nelson, B., Woolley, J., Pantelis, C., McGuire, P. \& Mechelli, A. (2014). Reduced parahippocampal cortical thickness in subjects at ultra-high risk for psychosis. Psychol Med 44, 489-98.

Trotta, A., Murray, R. M. \& Fisher, H. L. (2015). The impact of childhood adversity on the persistence of psychotic symptoms: a systematic review and meta-analysis. Psychol Med 45, 2481-98.

van den Berg, D. P., de Bont, P. A., van der Vleugel, B. M., De Roos, C., de Jongh, A., van Minnen, A. \& van der Gaag, M. (2015). Trauma-focused treatment in PTSD patients with psychosis: symptom exacerbation, adverse events, and revictimization. Schizophrenia bulletin 42, 693-702.

van den Berg, D. P., van der Vleugel, B. M., de Bont, P. A., Staring, A. B., Kraan, T., Ising, H., de Roos, C., de Jongh, A., van Minnen, A. \& van der Gaag, M. (2016). Predicting traumafocused treatment outcome in psychosis. Schizophrenia research 176, 239-244.

van Os, J., Reininghaus, U. \& Meyer-Lindenberg, A. (2016). The Search for Environmental Mechanisms Underlying the Expression of Psychosis: Introduction. Schizophr Bull. 
van Os, J., Rutten, B. P., Myin-Germeys, I., Delespaul, P., Viechtbauer, W., van Zelst, C., Bruggeman, R., Reininghaus, U., Morgan, C., Murray, R. M., Di Forti, M., McGuire, P., Valmaggia, L. R., Kempton, M. J., Gayer-Anderson, C., Hubbard, K., Beards, S., Stilo, S. A., Onyejiaka, A., Bourque, F., Modinos, G., Tognin, S., Calem, M., O'Donovan, M. C., Owen, M. J., Holmans, P., Williams, N., Craddock, N., Richards, A., Humphreys, I., Meyer-Lindenberg, A., Leweke, F. M., Tost, H., Akdeniz, C., Rohleder, C., Bumb, J. M., Schwarz, E., Alptekin, K., Ucok, A., Saka, M. C., Atbasoglu, E. C., Guloksuz, S., Gumus-Akay, G., Cihan, B., Karadag, H., Soygur, H., Cankurtaran, E. S., Ulusoy, S., Akdede, B., Binbay, T., Ayer, A., Noyan, H., Karadayi, G., Akturan, E., Ulas, H., Arango, C., Parellada, M., Bernardo, M., Sanjuan, J., Bobes, J., Arrojo, M., Santos, J. L., Cuadrado, P., Rodriguez Solano, J. J., Carracedo, A., Garcia Bernardo, E., Roldan, L., Lopez, G., Cabrera, B., Cruz, S., Diaz Mesa, E. M., Pouso, M., Jimenez, E., Sanchez, T., Rapado, M., Gonzalez, E., Martinez, C., Sanchez, E., Olmeda, M. S., de Haan, L., Velthorst, E., van der Gaag, M., Selten, J. P., van Dam, D., van der Ven, E., van der Meer, F., Messchaert, E., Kraan, T., Burger, N., Leboyer, M., Szoke, A., Schurhoff, F., Llorca, P. M., Jamain, S., Tortelli, A., Frijda, F., Vilain, J., Galliot, A. M., Baudin, G., Ferchiou, A., Richard, J. R., Bulzacka, E., Charpeaud, T., Tronche, A. M., De Hert, M., van Winkel, R., Decoster, J., Derom, C., Thiery, E., Stefanis, N. C., Sachs, G., Aschauer, H., Lasser, I., Winklbaur, B., Schlogelhofer, M., Riecher-Rossler, A., Borgwardt, S., Walter, A., Harrisberger, F., Smieskova, R., Rapp, C., Ittig, S., Soguel-dit-Piquard, F., Studerus, E., Klosterkotter, J., Ruhrmann, S., Paruch, J., Julkowski, D., Hilboll, D., Sham, P. C., Cherny, S. S., Chen, E. Y., Campbell, D. D., Li, M., Romeo-Casabona, C. M., Emaldi Cirion, A., Urruela Mora, A., Jones, P., Kirkbride, J., Cannon, M., Rujescu, D., Tarricone, I., Berardi, D., Bonora, E., Seri, M., Marcacci, T., Chiri, L., Chierzi, F., Storbini, V., Braca, M., Minenna, M. G., Donegani, I., Fioritti, A., La Barbera, D., La Cascia, C. E., Mule, A., Sideli, L., Sartorio, R., Ferraro, L., Tripoli, G., Seminerio, F., Marinaro, A. M., McGorry, P., Nelson, B., Amminger, G. P., Pantelis, C., Menezes, P. R., Del-Ben, C. M., Gallo Tenan, S. H., Shuhama, R., Ruggeri, M., Tosato, S., Lasalvia, A., Bonetto, C., Ira, E., Nordentoft, M., Krebs, M. O., Barrantes-Vidal, N., Cristobal, P., Kwapil, T. R., Brietzke, E., Bressan, R. A., Gadelha, A., Maric, N. P., Andric, S., Mihaljevic, M. \& Mirjanic, T. (2014). Identifying gene-environment interactions in schizophrenia: contemporary challenges for integrated, large-scale investigations. Schizophr Bull 40, 729-36.

Varese, F., Smeets, F., Drukker, M., Lieverse, R., Lataster, T., Viechtbauer, W., Read, J., van Os, J. \& Bentall, R. P. (2012). Childhood adversities increase the risk of psychosis: a metaanalysis of patient-control, prospective- and cross-sectional cohort studies. Schizophr Bull 38, 661-71.

Velthorst, E., Nieman, D. H., Linszen, D., Becker, H., de Haan, L., Dingemans, P. M., Birchwood, M., Patterson, P., Salokangas, R. K., Heinimaa, M., Heinz, A., Juckel, G., von Reventlow, H. G., French, P., Stevens, H., Schultze-Lutter, F., Klosterkotter, J. \& Ruhrmann, S. (2010). Disability in people clinically at high risk of psychosis. Br J Psychiatry 197, 278-84.

Vijayakumar, N., Mills, K. L., Alexander-Bloch, A., Tamnes, C. K. \& Whittle, S. (2018). Structural brain development: A review of methodological approaches and best practices. Dev Cogn Neurosci 33, 129-148.

Wechsler, D. (1981). Manual for the adult intelligence scale-revised. New York: Psychological Corporation.

Wechsler, D. (1991). WISC-III Wechsler Intelligence Scale for Children-third edition: Manual. San Antonio, TX: The Psychological Corporation.

Wechsler, D. (1999). Wechsler abbreviated scale of intelligence. Psychological Corporation. Yung, A. R., Cotter, J., Wood, S. J., McGorry, P., Thompson, A. D., Nelson, B. \& Lin, A. (2015). Childhood maltreatment and transition to psychotic disorder independently predict long-term functioning in young people at ultra-high risk for psychosis. Psychol Med 45, 3453-65. 
Yung, A. R. \& McGorry, P. D. (1996). The initial prodrome in psychosis: descriptive and qualitative aspects. Aust N Z J Psychiatry 30, 587-599.

Yung, A. R., Nelson, B., McGorry, P. D., Wood, S. J. \& Lin, A. (2019). Persistent negative symptoms in individuals at Ultra High Risk for psychosis. Schizophrenia research 206, 355-361. Yung, A. R., Phillips, L. J., Yuen, H. P., Francey, S. M., McFarlane, C. A., Hallgren, M. \& McGorry, P. D. (2003). Psychosis prediction: 12-month follow up of a high-risk ("prodromal") group. Schizophrenia Research 60, 21-32.

Yung, A. R., Phillips, L. J., Yuen, H. P. \& McGorry, P. D. (2004). Risk factors for psychosis in an ultra high-risk group: psychopathology and clinical features. Schizophrenia Research 67, 131142.

Yung, A. R., Yuen, H. P., McGorry, P. D., Phillips, L. J., Kelly, D., Dell'Olio, M., Francey, S. M., Cosgrave, E. M., Killackey, E., Stanford, C., Godfrey, K. \& Buckby, J. (2005). Mapping the onset of psychosis: The comprehensive assessment of at-risk mental states. Australian and New Zealand Journal of Psychiatry 39, 964-971.

Ziermans, T. B., Schothorst, P. F., Schnack, H. G., Koolschijn, P. C., Kahn, R. S., van Engeland, H. \& Durston, S. (2012). Progressive structural brain changes during development of psychosis. Schizophr Bull 38, 519-30.

\section{Figure Legends}


Figure 1. Regions of Interest. Freesurfer parcellated regions chosen for the current study. Cortical thickness and surface area was calculated for each region in each hemisphere.

Figure 2. Cortical thickness as a mediator. The Right Middle Temporal Gyrus acted as a significant mediator for the effects childhood sexual trauma had on transition to psychosis. Mean standardized path coefficients are presented with their associated $t$ statistic (coefficient divided by its standard error). CI- Confidence interval. Transition status was coded as No (0) and Yes (1) -see methods. Significance was set at $\alpha=0.05$.

\section{Tables}

Table 1. Characteristics of Participants at Baseline

\section{Supplementary Material}

Supplementary Table 1. Relationship between cortical thickness regions of interest (ROI/s) and Sexual Abuse and Transition to psychosis

Supplementary Table 2. Relationship between surface area regions of interest (ROI/s) and Sexual Abuse and Transition to psychosis

Supplementary Table 3. Relationship between cortical thickness regions of interest (ROI/s) and Physical Abuse

Supplementary Table 4. Relationship between surface area regions of interest (ROI/s) and Physical Abuse 
Supplementary Table 5. Relationship between cortical thickness regions of interest (ROI/s) and Emotional Abuse

Supplementary Table 6. Relationship between surface area regions of interest (ROI/s) and Emotional Abuse

Supplementary Table 7. Relationship between cortical thickness regions of interest (ROI/s) and Physical Neglect

Supplementary Table 8. Relationship between surface area regions of interest (ROI/s) and Physical Neglect

Supplementary Table 9. Relationship between cortical thickness regions of interest (ROI/s) and Emotional Neglect

Supplementary Table 10. Relationship between surface area regions of interest (ROI/s) and Emotional Neglect 


\section{University Library}

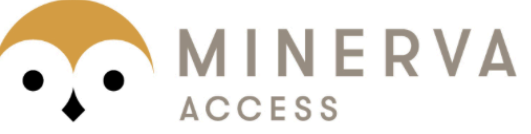

A gateway to Melbourne's research publications

Minerva Access is the Institutional Repository of The University of Melbourne

Author/s:

Rapado-Castro, M;Whittle, S;Pantelis, C;Thompson, A;Nelson, B;Ganella, EP;Lin, A;Reniers, RLEP;McGorry, PD;Yung, AR;Wood, SJ;Bartholomeusz, CF

Title:

Does cortical brain morphology act as a mediator between childhood trauma and transition to psychosis in young individuals at ultra-high risk?

Date:

2020-10-01

Citation:

Rapado-Castro, M., Whittle, S., Pantelis, C., Thompson, A., Nelson, B., Ganella, E. P., Lin, A., Reniers, R. L. E. P., McGorry, P. D., Yung, A. R., Wood, S. J. \& Bartholomeusz, C. F. (2020). Does cortical brain morphology act as a mediator between childhood trauma and transition to psychosis in young individuals at ultra-high risk?. SCHIZOPHRENIA RESEARCH, 224, pp.116-125. https://doi.org/10.1016/j.schres.2020.09.017.

Persistent Link:

http://hdl.handle.net/11343/250285 